M.I.T Media Laboratory Perceptual Computing Section Technical Report No. 482

Appeared: Proceedings of the International Conference on Acoustics, Speech, and Signal Processing

Phoenix, Arizona, March 15-19, 1999

\title{
A Spectral 2-D Wold Decomposition Algorithm for Homogeneous Random Fields
}

\author{
Fang Liu \\ MIT Lincoln Laboratory \\ 244 Wood St., Lexington, MA 02173 \\ fliu@ll.mit.edu
}

\begin{abstract}
The theory of the 2- $D$ Wold decomposition of homogeneous random fields is effective in image and video analysis, synthesis, and modeling. However, a robust and computationally efficient decomposition algorithm is needed for use of the theory in practical applications. This paper presents a spectral 2- $D$ Wold decomposition algorithm for homogeneous and near homogeneous random fields. The algorithm relies on the intrinsic fundamental-harmonic relationship among Fourier spectral peaks to identify harmonic frequencies, and uses a Hough transformation to detect spectral evanescent components. A local variance based procedure is developed to determine the spectral peak support. Compared to the two other existing methods for Wold decompositions, global thresholding and maximumlikelihood parameter estimation, this algorithm is more robust and flexible for the large variety of natural images, as well as computationally more efficient than the maximum-likelihood method.
\end{abstract}

\section{Introduction}

Characterizing the relatively homogeneous texture regions in image and video data has always been an important research area, with applications ranging from segmentation and coding to pattern matching and recognition. In new applications such as content-based access of digital libraries, texture is one of the most commonly used low-level features.

A textured image region can often be regarded as a homogeneous (stationary) random field. The twodimensional (2-D) Wold-like decomposition theory for homogeneous random fields has previously been introduced to texture analysis and synthesis in still images [1] and to periodic motion detection and segmentation in video [2]. The 2-D Wold theory allows an image pattern to be decomposed into three mutually orthogonal components. The perceptual characteristics of these components can be described as "periodicity", "directionality", and "randomness", agreeing closely with the dimensions of human

*This work was supported in part by IBM and NEC, and was conducted at the MIT Media Laboratory. The writing was supported in part by DARPA under contract \#F19628-95-C-0002.

\author{
Rosalind W. Picard \\ MIT Media Laboratory \\ 20 Ames St., Cambridge, MA 02139 \\ picard@media.mit.edu
}

texture perception identified in [3]. It was shown in [4], compared to other state-of-the-art texture models, Woldbased modeling offers perceptually more satisfying results in image database retrieval. Furthermore, demonstrated recently via a human study [5], the Wold component energy provides a good computational measure for the top dimension of human texture perception, the dimension of repetitiveness vs. randomness.

A main challenge in Wold-based image modeling is to develop an efficient and robust 2-D Wold decomposition algorithm. To date, the algorithms reported are either computationally prohibitive for practical applications or not robust enough to handle the large variety of natural images. This paper presents a new decomposition algorithm that is computationally efficient and robust for natural image data.

\section{2-D Wold Decomposition}

Consider a homogeneous and regular random field $\{y(m, n)\},(m, n) \in \mathcal{Z}^{2}$. The 2-D Wold decomposition allows the field to be decomposed into two mutually orthogonal components [6]:

$$
y(m, n)=v(m, n)+w(m, n)
$$

where $\{v(m, n)\}$ is deterministic and $\{w(m, n)\}$ is indeterministic. The deterministic component can be further decomposed into the mutually orthogonal harmonic component $\{h(m, n)\}$ and evanescent component $\{g(m, n)\}$ :

$$
v(m, n)=h(m, n)+g(m, n) .
$$

In the frequency domain, the spectral distribution function (SDF) of $\{y(m, n)\}$ can be uniquely represented by the SDF's of its component fields:

$$
F_{y}(\xi, \eta)=F_{v}(\xi, \eta)+F_{w}(\xi, \eta)
$$

where $F_{v}(\xi, \eta)=F_{h}(\xi, \eta)+F_{g}(\xi, \eta)$, and functions $F_{h}(\xi, \eta)$ and $F_{g}(\xi, \eta)$ correspond to spectral singularities supported by point-like and line-like regions, respectively. Examples of natural textures containing different prominent Wold components are shown in Figure 1.

\section{Previous Work}

Two decomposition methods have been proposed in the literature [7] [1]. The first is a maximum-likelihood direct parameter estimation procedure, which provides parametric descriptions of image Wold components. Its developers reported that the algorithm can be computationally expensive, especially when the number of spectral peaks is 


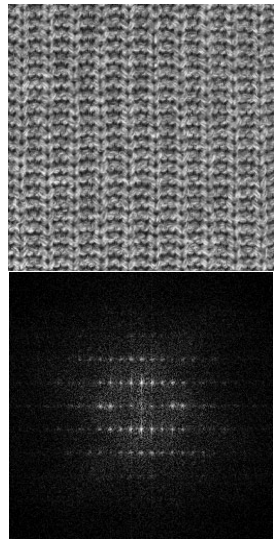

(a)

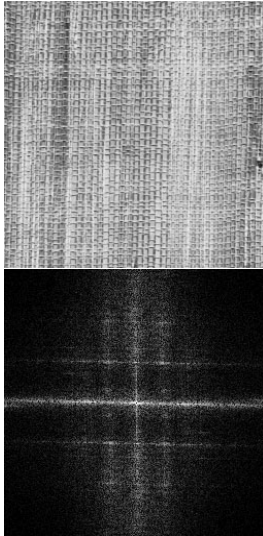

(b)

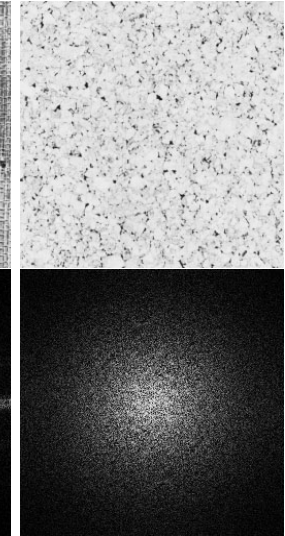

(c)
Figure 1: Examples of natural textures with different prominent Wold components. Top row: originals. Bottom row: Fourier magnitudes. (a) Sweater, with a strong harmonic component (spectral peaks supported by point-like regions). (b) Brodatz texture D78, with a strong evanescent component (spectral peaks supported by line-like regions). (c) Brodatz texture D32, with mostly an indeterministic component (relatively smooth spectrum).

large or the energy in the spectral peaks is not very high compared to that in the neighboring Fourier frequencies [7]. Unfortunately, these situations often arise in natural images. The second method is a spectral decomposition procedure [1]. It applies a global threshold to the image periodogram, and the Fourier frequencies with magnitude values larger than the threshold are considered to be the harmonic or the evanescent components. Although this method is computationally efficient, it is not robust enough for the large variety of natural texture patterns. As shown in Figure 2, the support region of a harmonic peak in a natural texture periodogram is usually not a point, but a small spread surrounding the central frequency. Therefore, two issues are essential for a decomposition scheme: locating the spectral peak central frequencies, and determining the peak support regions. In the example in Figure 2, the Brodatz texture [8] D11 has some high frequency spectral peaks that are only locally large in magnitude. Global thresholding yields either poor segmentation of the peak supports as in Figure 2 (c) or inaccurate peak identification as in Figure 2 (d). In natural textures, this type of spectra abounds. Hence, a new algorithm is needed.

\section{New Decomposition Algorithm}

The new algorithm takes a spectral decomposition approach. It decomposes an image by extracting its Fourier spectral peaks supported by point-like and line-like regions. As discussed before, a spectral approach needs to locate the peak central frequencies as well as to estimate the peak supports. Hence the new algorithm consists of four parts: harmonic peak detection, evanescent line detection, peak support estimation, and decomposition.

\subsection{Harmonic Peak Detection}

The harmonic peaks are detected in the Fourier magnitude image. The quadrants of the image are rearranged such

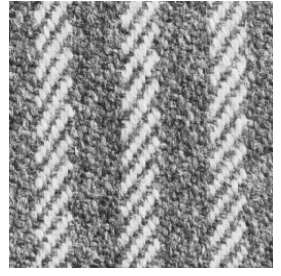

(a)

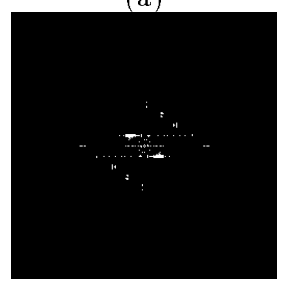

(c)

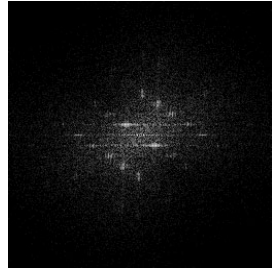

(b)

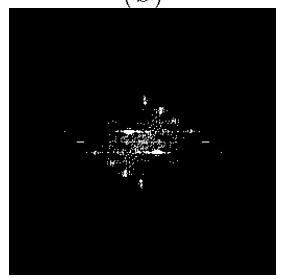

(d)
Figure 2: Spectral peak detection of Brodatz texture D11 using global thresholding. (a) Original. (b) Fourier magnitudes. (c) A high threshold gives poor estimates of peak support. (d) A low threshold gives better peak support but picks up indeterministic frequencies. See Figure 8 (a) for results from the new algorithm.

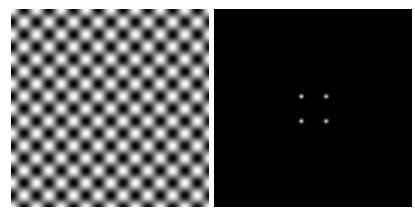

(a)

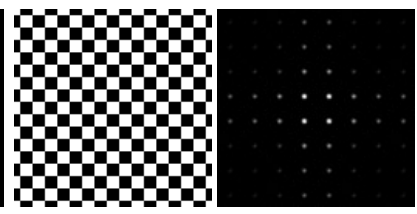

(b)
Figure 3: Fundamental-harmonic relationship among harmonic peaks. (a) 2-D sine grating and its Fourier magnitudes, having two frequencies. (b) Checkerboard pattern and its Fourier magnitudes, having two fundamentals (with the same frequencies as in (a)) and a series of harmonics. Edges in natural patterns usually fall in between these extreme cases, hence the fundamental-harmonic relationship usually exists.

that the zero frequency is at the center. To reduce the edge effect, a Gaussian taper can be used when computing the image Fourier transforms. Since for real images the magnitude values are symmetric to the zero frequency, only half of the frequency plane needs to be considered.

As shown previously, spectral peaks can be locally but not globally large. Hence, local maxima are first found in the magnitude image using a $5 \times 5$ neighborhood. Values below $5 \%$ of the entire magnitude range are ignored. To identify the true harmonic peaks from the local maxima, the intrinsic fundamental-harmonic relationship among the harmonic peaks is used. This relationship is illustrated in Figure 3. A local maximum qualifies as a harmonic peak only when its frequency is either a fundamental or a harmonic. A fundamental is a frequency that can be used to linearly express the frequencies of some other local maxima. A harmonic is a frequency that can be represented as a linear combination of some fundamentals.

Due to the sampling effect of discrete Fourier transforms, the frequency samples may not align to the true peaks of the continuous spectrum. Small sampling errors 

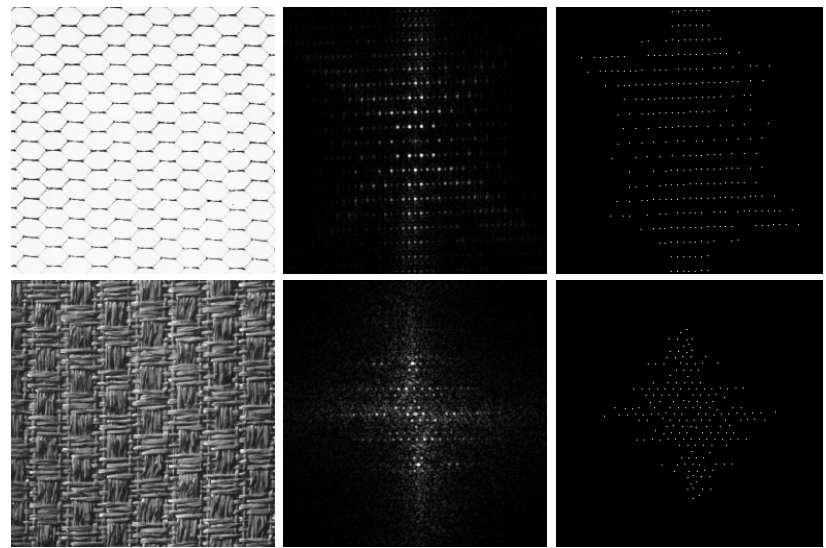

Figure 4: Spectral harmonic peak detection on Brodatz texture D34 (top row) and D82 (bottom row). Left: original. Center: Fourier magnitudes. Right: detected harmonic peaks.

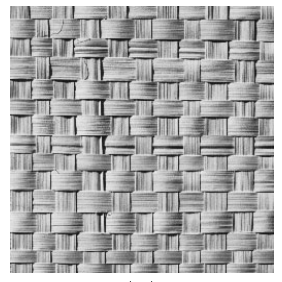

(a)

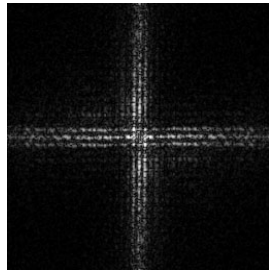

(b)

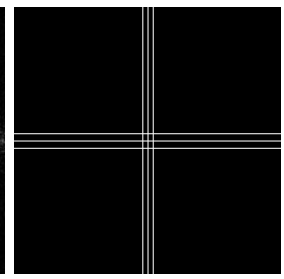

(c)

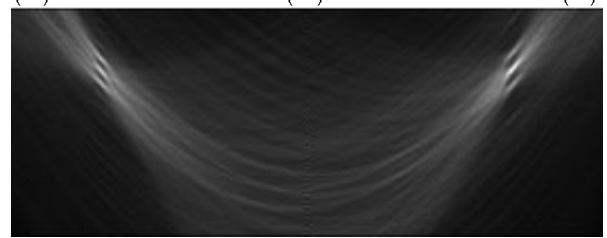

(d)

Figure 5: Example of spectral evanescent line detection. (a) Brodatz texture D64. (b) Fourier magnitudes of (a). (c) Lines found in (b). (d) Hough transform of (b). Lines in (c) correspond to the six large local maxima in (d).

in the fundamentals can cause misidentification of their high frequency harmonics. To reduce the sampling effect, each fundamental frequency value is refined to subsample precision using the harmonics found at the multiples of the fundamental, and a tolerance of two sample points in both row and column directions is used for frequency matching.

Examples of harmonic peak detection are shown in Figure 4.

\subsection{Evanescent Line Detection}

The Hough transformation method for line detection [9] is used to detect the evanescent lines in the Fourier magnitude images. Prior to applying the Hough transform, any large spectral values associated with the harmonic peaks should be removed. An example of evanescent line detection is shown in Figure 5, where six lines are found for the Brodatz texture D64.
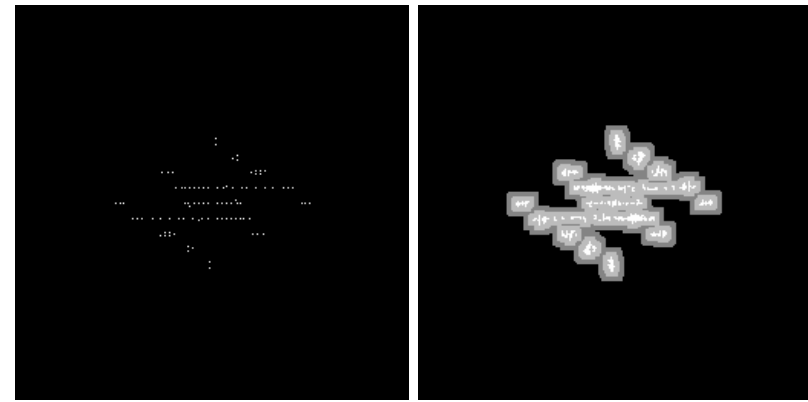

Figure 6: Left: spectral harmonic peaks detected in Brodatz texture D11. Right: estimated peak support (white) and the corresponding adjacent (light gray) and ambient (dark gray) areas after 4 iterations. The original and its Fourier magnitude image are in Figure 2.

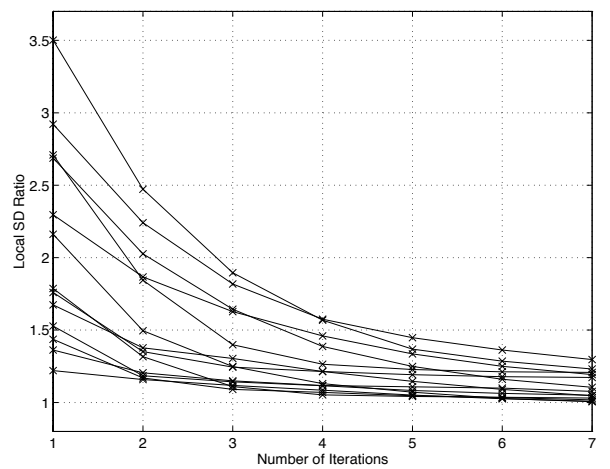

Figure 7: Fourier magnitude local standard deviation ratios of 13 Brodatz textures in the first 7 iterations.

\subsection{Peak Support Estimation}

An iterative procedure is used to estimate the spectral peak support regions in a Fourier magnitude image. The process is initialized by the detected harmonic peaks and evanescent lines. Along the lines, frequencies with magnitude values less than $5 \%$ of the magnitude range are omitted. At the beginning of each iteration, a 2-D Gaussian surface is fitted to the magnitude image to coarsely model the relatively smooth "background". Based on the local variance of the fitting residual, new support frequencies are identified and removed, and the magnitude image becomes "smoother". This smoothness is measured by the ratio between the averaged local standard deviations $(5 \times 5$ estimation window) in the areas adjacent to the estimated peak support regions and that in the ambient areas. Figure 6 illustrates the technique and Figure 7 shows how the local standard deviation ratio reduces after each iteration. The iterative process terminates when the change of the local standard deviation ratio between two iterations is less than 0.1 . Further details of this iterative procedure can be found in [5].

\subsection{Decomposition}

The final decomposition of a homogeneous random field is based on the decomposition of its spectral Wold components. Denote the image's 2-D discrete Fourier transform (DFT) as $Y(k, l)$, the corresponding frequency plane as $\mathcal{D}$, and the set of spectral peak and peak support frequencies 


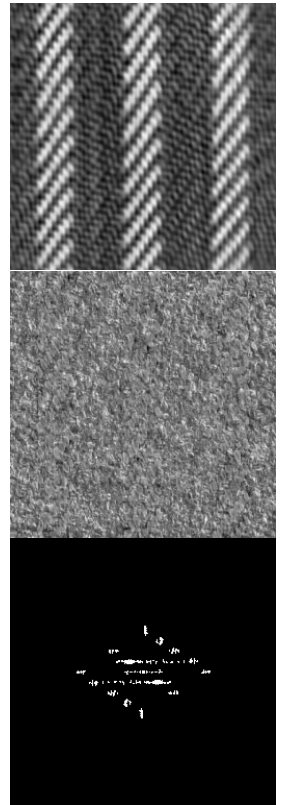

(a)

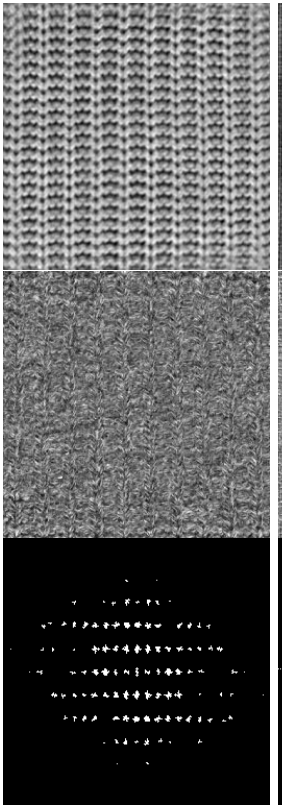

(b)

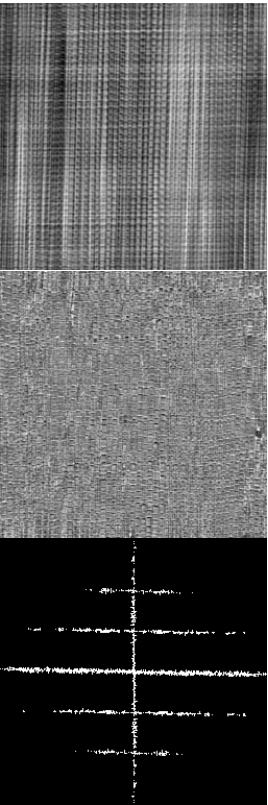

(c)
Figure 8: Decomposition of (a) Brodatz texture D11, (b) Sweater pattern, and (c) Brodatz texture D78. The originals and their Fourier magnitudes are in Figure 1 and Figure 2. Top row: harmonic or evanescent components. Middle row: indeterministic components. Bottom row: identified spectral peak frequencies.

identified in the previous stages as $\mathcal{D}_{v}$. The DFT of the random field can be then decomposed into the deterministic component

$$
V(k, l)= \begin{cases}Y(k, l), & (k, l) \in \mathcal{D}_{v} \\ 0, & (k, l) \in \mathcal{D},(k, l) \notin \mathcal{D}_{v}\end{cases}
$$

and the indeterministic component

$$
W(k, l)= \begin{cases}Y(k, l), & (k, l) \in \mathcal{D},(k, l) \notin \mathcal{D}_{v} \\ 0, & (k, l) \in \mathcal{D}_{v}\end{cases}
$$

The deterministic field $v(m, n)$ and the indeterministic field $w(m, n)$ are obtained by computing the inverse DFT of $V(k, l)$ and $W(k, l)$, respectively. Note that, when necessary, the deterministic component can be further decomposed into the harmonic and the evanescent components in a similar manner.

\subsection{Examples}

Three decomposition examples are shown in Figure 8. The originals, shown in Figure 1 and Figure 2, have size $256 \times$ 256. The computing time ranges from 15 to 20 seconds on a HP9000/735 Workstation.

\section{Conclusions}

A spectral 2-D Wold decomposition algorithm for homogeneous and near homogeneous random fields is presented. This algorithm relies on the intrinsic fundamentalharmonic relationship among image Fourier spectral peaks to identify harmonic frequencies, and uses a Hough transformation to detect spectral evanescent components. A local variance based procedure is developed to determine the spectral peak support. Compared to the two other existing Wold decomposition algorithms, the global thresholding and the maximum-likelihood parameter estimation, this algorithm is more robust and flexible for the large variety of natural images, as well as computationally more efficient than the maximum-likelihood method.

\section{References}

[1] J. M. Francos, A. Zvi Meiri, and B. Porat. A unified texture model based on a 2-D Wold-like decomposition. IEEE T. Sig. Proc., pages 2665-2678, August 1993.

[2] F. Liu and R. W. Picard. Finding periodicity in space and time. In Proc. Int. Conf. on Computer Vision, pages 376-383, Bombay, India, January 4-7 1998.

[3] A. R. Rao and G. L. Lohse. Towards a texture naming system: identifying relevant dimensions of texture. Vision Research, 36(11):1649-1669, 1996.

[4] F. Liu and R. W. Picard. Periodicity, directionality, and randomness: Wold features for image modeling and retrieval. IEEE T. Pat. Analy. and Machine Intel., 18(7):722-733, July 1996.

[5] F. Liu. Modeling Spatial and Temporal Textures. PhD thesis, Massachusetts Institute of Technology, 1997.

[6] J. M. Francos. Orthogonal decompositions of 2-D random fields and their applications in 2-D spectral estimation. In N. K. Bose and C. R. Rao, editors, Signal Processing and Its Applications, Handbook of Statistics, Vol. 10, pages 207-227. North Holland, 1993.

[7] J. M. Francos, A. Narasimhan, and J. W. Woods. Maximum likelihood parameter estimation of discrete homogeneous random fields with mixed spectral distributions. IEEE T. Sig. Proc., 44(5):1242-1255, May 1996.

[8] P. Brodatz. Textures: A Photographic Album for Artists and Designers. Dover, New York, 1966.

[9] R. M. Haralick and L. G. Shapiro. Computer and Robot Vision, volume 1. Addison-Wesley Publishing Company, Inc., 1992. 